\title{
Detection of Elliptical Traffic Signs
}

\author{
Manal El Baz ${ }^{(凶)}(\mathbb{D}$, Taher Zaki(D), and Hassan Douzi (D) \\ Image and Pattern Recognition - Intelligent and Communicating Systems Laboratory, \\ Faculty of Sciences, Ibn Zohr University, Agadir, Morocco \\ elbazmanal@gmail.com
}

\begin{abstract}
Detection of elliptical features is a challenging and important task in computer vision. In fact, ellipses can describe many objects in real images like manufactured objects, cells, ball, or traffic signs. Furthermore, an ellipse is defined by five parameters: the center coordinates, the semi major axe, the semi minor axe and the orientation, which require more computational power to estimate them. Some non-ideal ellipses cause also difficulties for detection such as occlusion, appearance of multiple ellipses at same time and non-parallel ellipse. In this paper, we are interested in detecting elliptical traffic signs. We present a method for detecting different cases of ellipses: simple, partially occluded, nonparallel, multiple ellipses in images. The method selects three lines to find the ellipse center, and then it calculates the value of the semi minor and major axes of the ellipse. Experiments show that the proposed method performs well on real images in the presence or not of noises.
\end{abstract}

Keywords: Detection of traffic signs $\cdot$ Ellipse detection - Binary image

\section{Introduction}

Detecting ellipse in an image has received attention by many researchers in recent decades [2-10]. Many methods are developed to detect an ellipse in an image. The most method used is the Hough transform. Firstly, Hough transform is invented in 1962 by Paul Hough [1] to detect straight lines in an image. Later, Hough transform is developed to detect other shapes such as ellipses [2-4]. An ellipse is a conic that is defined by five parameters: the semi major axe, the semi minor axe, the center and the orientation. The standard Hough transform and its derivatives calculate those parameters to extract an ellipse in an image. Teng et al. [2] use two-level voting algorithms to estimate the five ellipse parameters. They developed the randomized Hough transform to eliminate noisy edge points dissimilar to ellipse patterns by analyzing the variation of the angles of tangential lines for edge points. Latour et al. [3] adopt the elliptical Hough transform to extract ellipses in an image using two edge points and their associated image gradients. Their method is based on algebraic framework that is expressed in the point form or the tangential form. Xie et al. [4] accumulate the length of ellipse minor axis by using one dimensional accumulator array. If the vote of 
minor axis is greater than the threshold, then the ellipse and its parameters are extracted.

In other hand, other methods for detecting ellipse; which are not based on Hough transform; are proposed: Ouellet et al.'s method [5] is based on the dual conic model. It exploits directly the raw gradient information in the neighborhood of an ellipse's boundary, and minimize the algebraic distance to constrain the dual conic to a dual ellipse. It exists also methods based on tracking arc segments and connectivity between edge pixels to detect ellipses. Kim et al. [6] extract arc segments and then merge them into an ellipse. Ellipses can be detected from the extended elliptical arc segments. Jia et al. [7] used a projective invariance of characteristic number to prune line segments and pick arc segments lying on an ellipse. The picked arc segments are used to estimate ellipse parameters. They determine the ellipse center by finding the algebraic average of coordinates of intersections of bisectors of 16 parallel chords of each arc segments lying on an ellipse. Libuda et al. [8] firstly extract short straight lines, then they merge these lines to small arcs which will be combined to extended arcs. Then, they create an ellipse by merging extended arcs. Mai et al.'s method [9] is also based on arc segments extraction. They use the hierarchical algorithm to detect ellipse in four stages: firstly, they extract line segments that will be linked to synthetic arc segments. Then, they fit ellipse in each arc group by using RANSAC.

In this paper, we present a new method for detecting elliptical traffic signs in an image. We use three lines to find the ellipse center. Two of those lines are parallels; therefore, the third goes through the middles of the two lines. The middle of the third line is the ellipse center. Hence, we calculate the semi major and minor of the detected ellipse. The result of the proposed method shows that it works better than the state-of-the-art ellipse detectors on detecting elliptical traffic signs: simple ellipse, partially occluded ellipse, non-parallel ellipse, multiple ellipses, and noisy image. This paper is organized as follow: firstly, we present our method in Sect. 2, then in Sect. 3, we discuss the result of our method and finally we make some conclusions in Sect. 4 .

\section{Method Description}

\subsection{Circle Detection}

At first of our work in shape detection development, we were interested in finding circles in images. We take 500 points in an image and then we relied on pairs of points to determine the circle center. We choose randomly two points: $P_{1}\left(x_{1}, y_{1}\right)$ and $P_{2}\left(x_{2}, y_{2}\right)$ in the image. The coordinates of circle center $\Omega(a, b)$ is:

$$
\begin{aligned}
& a=\frac{x_{1}+x_{2}}{2} . \\
& b=\frac{y_{1}+y_{2}}{2} .
\end{aligned}
$$


and the circle radius is:

$$
r=\frac{\sqrt{\left(x_{1}-x_{2}\right)^{2}+\left(y_{1}-y_{2}\right)^{2}}}{2} .
$$

We calculate the number of points that satisfy the Cartesian equation of each circle:

$$
(x-a)^{2}+(y-b)^{2}=r^{2} .
$$

We consider NbMax as the maximum of points that satisfy a Cartesian equation of a circle. If more than half of NbMax of points are belonging to one circle in the image, we extract the present circle. The result of this method shows that it does not detect circles correctly and the false positive rate is higher (we got $29,56 \%$ as F-measure). Therefore, we decide to change our purpose: detecting ellipses rather than circles and defining lines to extract the center.

\subsection{Ellipse Detection}

Our method is about finding the ellipse center by three lines $\left(D_{1}\right),\left(D_{2}\right)$ and $\left(D_{3}\right)$ that intersect the ellipse. Two of those lines $\left(D_{1}\right)$ and $\left(D_{2}\right)$ are parallels. However, the third line $\left(D_{3}\right)$ goes through the middles of intersections of the two lines and the ellipse. The middle of intersections of $\left(D_{3}\right)$ and the ellipse is the ellipse center (see Fig. 1).

We consider two parallel lines $\left(D_{1}\right)$ and $\left(D_{2}\right)$, and the points $P_{1}\left(x_{1}, y_{1}\right), P_{2}\left(x_{2}, y_{2}\right)$ and $P_{3}\left(x_{3}, y_{3}\right), P_{4}\left(x_{4}, y_{4}\right)$ are respectively intersections of $\left(D_{1}\right)$ and $\left(D_{2}\right)$ and the ellipse. Equations of $\left(D_{1}\right)$ and $\left(D_{2}\right)$ are:

$$
\begin{aligned}
& \forall m_{1}, k_{1} \in \mathbb{R} \quad D_{1}: y=m_{1} x+k_{1} . \\
& \forall m_{2}, k_{2} \in \mathbb{R} \quad D_{2}: y=m_{2} x+k_{2} .
\end{aligned}
$$

We have:

$$
D_{1} \| D_{2} \quad \Leftrightarrow \quad m_{1}=m_{2} .
$$

and we have $P_{1}, P_{2} \in D_{1}$ and $P_{3}, P_{4} \in D_{2}$ so:

$$
\begin{gathered}
\left\{\begin{array} { l } 
{ y _ { 1 } = m _ { 1 } x _ { 1 } + k _ { 1 } } \\
{ y _ { 2 } = m _ { 1 } x _ { 2 } + k _ { 1 } }
\end{array} \Rightarrow \left\{\begin{array}{r}
m_{1}=\frac{y_{2}-y_{1}}{x_{2}-x_{1}} \\
k_{1}=y_{1}-m_{1} x_{1}
\end{array}\right.\right. \\
\left\{\begin{array} { l } 
{ y _ { 3 } = m _ { 2 } x _ { 3 } + k _ { 2 } } \\
{ y _ { 4 } = m _ { 2 } x _ { 4 } + k _ { 2 } }
\end{array} \Rightarrow \left\{\begin{array}{r}
m_{2}=\frac{y_{4}-y_{3}}{x_{4}-x_{3}} \\
k_{2}=y_{3}-m_{2} x_{3}
\end{array}\right.\right.
\end{gathered}
$$

The middle of $\left[P_{1} P_{2}\right]$ is the point $M_{1}\left(x_{M_{1}}, y_{M_{1}}\right)$ where:

$$
\begin{aligned}
& x_{M_{1}}=\frac{x_{1}+x_{2}}{2} . \\
& y_{M_{1}}=\frac{y_{1}+y_{2}}{2} .
\end{aligned}
$$


And the middle of $\left[P_{3} P_{4}\right]$ is the point $M_{2}\left(x_{M_{2}}, y_{M_{2}}\right)$ where:

$$
\begin{aligned}
& x_{M_{2}}=\frac{x_{3}+x_{4}}{2} . \\
& y_{M_{2}}=\frac{y_{3}+y_{4}}{2} .
\end{aligned}
$$

We consider $\left(D_{3}\right)$ goes through $M_{1}$ and $M_{2}$ so the equation of $\left(D_{3}\right)$ is as follow:

$$
\forall m_{3}, k_{3} \in \mathbb{R} \quad D_{3}: y=m_{3} x+k_{3} .
$$

where:

$$
m_{3}=\frac{y_{M_{2}}-y_{M_{1}}}{x_{M_{2}}-x_{M_{1}}} \quad \text { and } \quad k_{3}=y_{M_{1}}-m_{3} x_{M_{1}} .
$$

We consider the points $P_{5}\left(x_{5}, y_{5}\right)$ and $P_{6}\left(x_{6}, y_{6}\right)$ are intersections of $\left(D_{3}\right)$ and the ellipse. The middle of $\left[P_{5} P_{6}\right]$ is the center of the ellipse $C\left(x_{C}, y_{C}\right)$ so:

$$
\begin{aligned}
& x_{C}=\frac{x_{5}+x_{6}}{2} . \\
& y_{C}=\frac{y_{5}+y_{6}}{2} .
\end{aligned}
$$

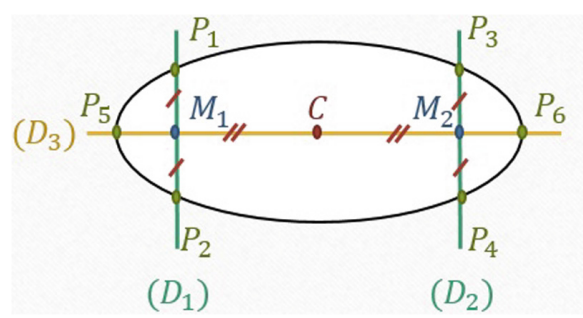

Fig. 1. The ellipse center

For finding semi major and minor axes of ellipse, we choose randomly four points $P_{1}^{\prime}\left(x_{P_{1}^{\prime}}, y_{P_{1}^{\prime}}\right), P_{2}^{\prime}\left(x_{P_{2}^{\prime}}, y_{P_{2}^{\prime}}\right), P_{3}^{\prime}\left(x_{P_{3}^{\prime}}, y_{P_{3}^{\prime}}\right)$ and $P_{4}^{\prime}\left(x_{P_{4}^{\prime}}, y_{P_{4}^{\prime}}\right)$ from the image where the ellipse center $C\left(x_{C}, y_{C}\right)$ is the middle of segments $\left[P_{1}^{\prime} P_{2}^{\prime}\right]$ and $\left[P_{3}^{\prime} P_{4}^{\prime}\right]$.

The values of a and $\mathrm{b}$ are respectively distance of $\left[P_{1}^{\prime} P_{2}^{\prime}\right]$ and $\left[P_{3}^{\prime} P_{4}^{\prime}\right]$ :

$$
\begin{aligned}
& a=\frac{\sqrt{\left(x_{P_{1}^{\prime}}-x_{P_{2}^{\prime}}\right)^{2}+\left(y_{P_{1}^{\prime}}-y_{P_{2}^{\prime}}\right)^{2}}}{2} . \\
& b=\frac{\sqrt{\left(x_{P_{3}^{\prime}}-x_{P_{4}^{\prime}}\right)^{2}+\left(y_{P_{3}^{\prime}}-y_{P_{4}^{\prime}}\right)^{2}}}{2} .
\end{aligned}
$$


$\mathrm{a}$ and $\mathrm{b}$ are respectively the semi major and minor axe of the ellipse if they satisfy the following conditions:

$$
\left\{\begin{array}{r}
0<b<a \\
0<\frac{\sqrt{a^{2}-b^{2}}}{a}<1 \\
\left(P_{1}^{\prime} P_{2}^{\prime}\right) \perp\left(P_{3}^{\prime} P_{4}^{\prime}\right)
\end{array}\right.
$$

The proposed method can be described in steps showed in Fig. 2.

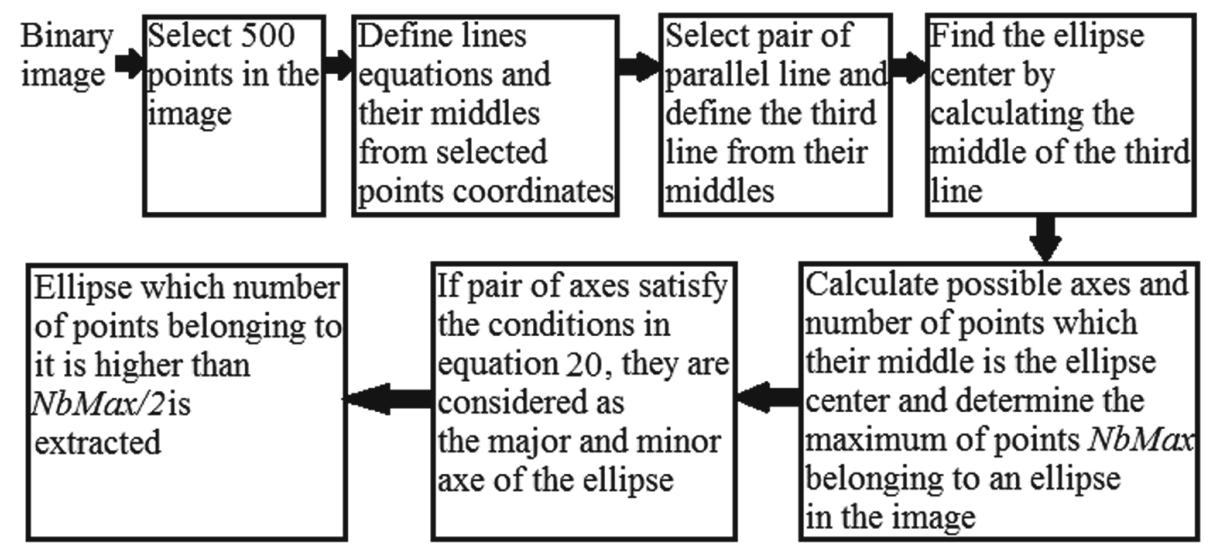

Fig. 2. Method description.

\section{Experimental Evaluation}

We experiment the methods on 69 images that contain different cases possible of ellipse: simple ellipse, partially occluded ellipse, non-parallel ellipse, multiple ellipses, noisy image; and its different size and position.

We use the following metrics to measure the performance of the methods:

$$
\begin{gathered}
\text { Precision }=\frac{T P}{T P+F P} . \\
\text { Recall }=\frac{T P}{T P+F N} . \\
F-\text { measure }=2 \times \frac{\text { Precision } \times \text { Recall }}{\text { Precision }+ \text { Recall }} .
\end{gathered}
$$


where:

TP: True positive.

FP: False positive.

FN: False negative.

Table 1. Result of methods.

\begin{tabular}{l|l|l|l|l}
\hline & Precision & \multicolumn{1}{|c|}{ Recall } & F-measure & Time \\
\hline Proposed method & $73.27 \%$ & $\mathbf{7 9 . 5 7 \%}$ & $\mathbf{7 6 . 2 9 \%}$ & $\mathbf{3 , 0 7} s$ \\
\hline ELSDc [10] & $\mathbf{7 3 . 5 6 \%}$ & $68.82 \%$ & $71.11 \%$ & $17,97 s$ \\
\hline Jia et al.'s method [7] & $57.55 \%$ & $65.59 \%$ & $61.31 \%$ & $4,92 s$ \\
\hline Xie et al.'s method [4] & $46.79 \%$ & $54.84 \%$ & $50.50 \%$ & $15.34 s$ \\
\hline
\end{tabular}

We compare our method to three popular state-of-the-art ellipse detectors: joint ellipse and line segment detector ELSDc proposed by Patraucean et al. [10], Jia et al.'s method [7] and Xie et al.'s method [4]. ELSDc [10] is a line segment and ellipse detector based on the contrario theory. This detector [10] performs accurately on synthetic and real images. ELSDc is available online at dev.ipol.im/ jirafa/elsdc/. Jia et al. method [7] is based on projective invariant to prune lines and pick out ellipses. It [7] proved its high performance in public datasets. This detector is shared online at https://github.com/dlut-dimt/ ellipse-detector. Xie et al.'s method [4] is an ellipse hough detection method. This method [4] is based on a one-dimensional accumulator array to vote on the minor axis. We reimplement Xie et al.'s method [4] according to the original paper. In experiments, we are interested on detecting elliptical traffic signs in images. Table 1 gives the result of the methods. It shows that our method has achieved higher recall and F-measure rate than the other methods. However, ELSDc performs better than our method in terms of precision. Furthermore, our method consumes lower computation time compared to ELSDc, Jia et al. method [7] and Xie et al.'s method [4]. ELSDc [10] suffers from large computation time because of the detection of line segments and ellipses all together.

The result of the proposed method on each case of ellipse is depicted in Fig. 3. The proposed method works well on all ellipse cases except multiple ellipses case. Its performance is poor if the image contains multiple ellipses. That happens because the false negative rate increases when more ellipses appear at the same time in an image (see Fig. 3 (f)). Moreover, our method has achieved good result on non-parallel ellipses. In contrast, the result that we obtained for ELSDc [10] and Xie et al.'s method [4] on non-parallel ellipses is lower than our method and Jia et al.'s method [7] in terms of F-measure: $78.26 \%$ for our method, $68.97 \%$ for Jia et al.'s method [7], $60.00 \%$ for Xie et al.'s method [4] and $50.00 \%$ for ELSDc [10]. 


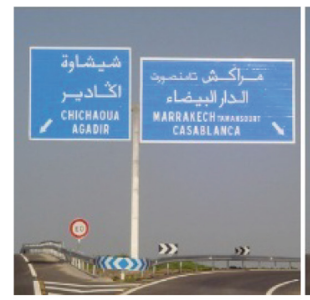

(a) Simple ellipse.

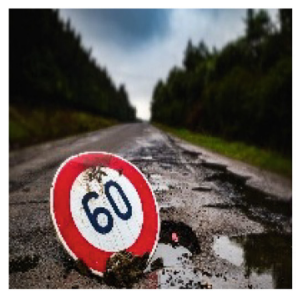

(c) Non-parallel and partially occluded ellipse.

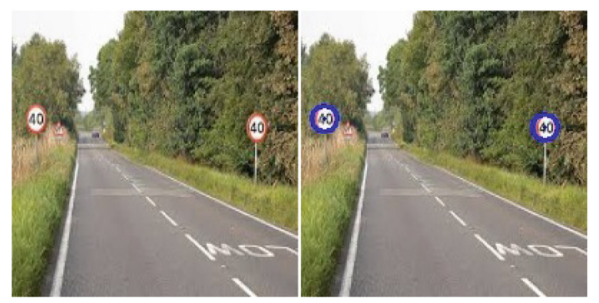

(e) Multiple ellipses.
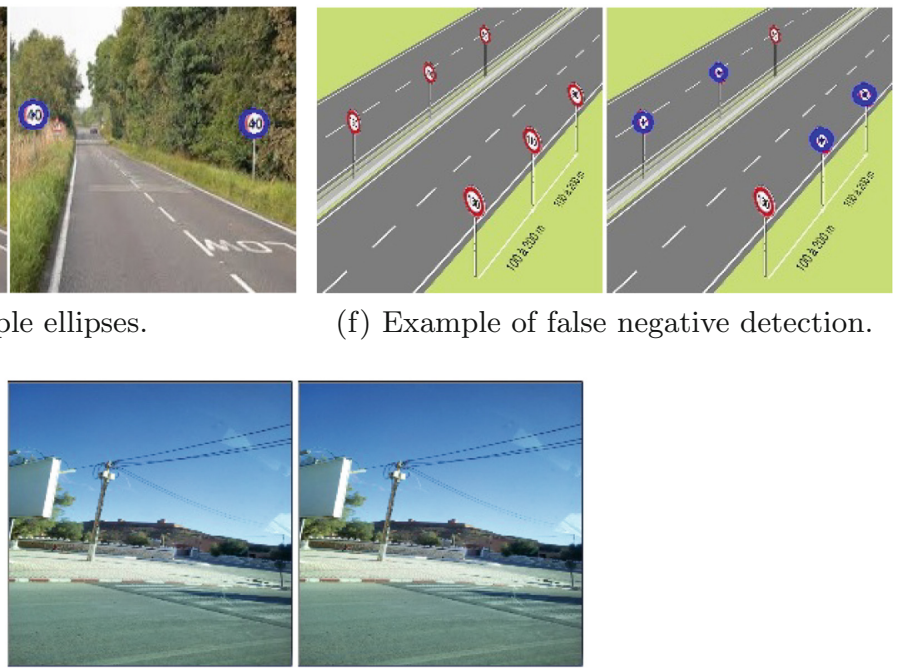

(f) Example of false negative detection.

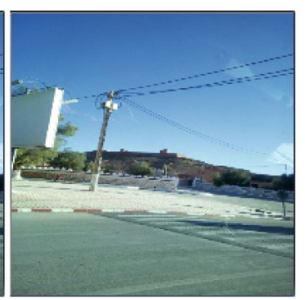

(g) Image without traffic sign.

Fig. 3. Elliptical traffic signs detection.

\section{Conclusion}

This paper has presented a method for detecting elliptical traffic signs in images. This method selects firstly two parallel lines, and then it determines the midpoints of segments limited by intersections of those two lines and the ellipse. The third line; which goes through the middles; is defined and its middle is the 
center of the researched ellipse. It has been shown that the proposed method can detect simple, partially occluded, non-parallel and multiple ellipses and it performs accurately on real images in presence or not of noises. Currently, we are developing the present method in order to increase its performance on images in which appear multiple ellipses.

\section{References}

1. Hough, P.V.C.: Method and means for recognizing complex patterns. U.S. Patent 3069654, Ser. No. 177156 Claims (1962)

2. Teng, Z., Kim, J.-H., Kang, D.-J.: Ellipse detection: a simple and precise method based on randomized Hough transform. Opt. Eng. 51(5), 057203-1-057203-14 (2012). https://doi.org/10.1117/1.OE.51.5.057203

3. Latour, P., Van Droogenbroeck, M.: Dual approaches for elliptic Hough transform: eccentricity/orientation vs center based. In: Couprie M., Cousty J., Kenmochi Y., Mustafa N. (eds.) Discrete Geometry for Computer Imagery. DGCI 2019. LNCS, vol. 11414, pp. 367-379. Springer, Charm (2019). https://doi.org/10.1007/978-3030-14085-4_29

4. Xie, Y., Ji, Q.: A new efficient ellipse detection method. In: International Conference on Pattern Recognition, vol. 2, pp. 957-960. IEEE, Quebec (2002). https:// doi.org/10.1109/ICPR.2002.1048464

5. Ouellet, J.-N., Hebert, P.: A simple operator for very precise estimation of ellipses. In: Fourth Canadian Conference on Computer and Robot Vision (CRV 2007), pp. 21-28. IEEE, Montreal (2007). https://doi.org/10.1109/CRV.2007.8

6. Kim, E., Haseyama, M., Kitajima, H.: Fast and robust ellipse extraction from complicated images. In: Proceedings of the IEEE International Conference on Information Technology and Applications (2002)

7. Jia, Q., Fan, X., Luo, Z., Song, L., Qiu, T.: A fast ellipse detector using projective invariant pruning. IEEE Trans. Image Process. 26(8), 3665-3679 (2017). https:// doi.org/10.1109/TIP.2017.2704660

8. Libuda, L., Grothues, I., Kraiss, K.-F.: Ellipse detection in digital image data using geometric features. In: Ranchordas, A., Araujo, H., Encarnaçao, B. (eds.) Proceedings of the First International Conference on Computer Vision Theory and Applications, vol. 1, pp. 175-180. SciTePress, Setubal (2006). https://doi.org/ 10.5220/0001362301750180

9. Mai, F., Hung, Y.S., Zhong, H., Sze, W.F.: A hierarchical approach for fast and robust ellipse extraction. In: 2007 IEEE International Conference on Image Processing. ICIP 2007, vol. 5, pp. V-345-V-348. IEEE, San Antonio (2007). https:// doi.org/10.1109/ICIP.2007.4379836

10. Patraucean, V., Gurdjos, P., Von Gioi, R.G.: Joint a contrario ellipse and line detection. IEEE Trans. Pattern Anal. Mach. Intell. 39(4), 788-802 (2017). https:// doi.org/10.1109/TPAMI.2016.2558150 